\title{
Le monde nouveau (pour moi) de l'hyperconnection
}

\section{Jean Martin}

Dr méd., membre de la rédaction

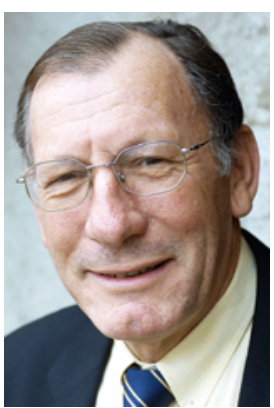

Je viens de passer une semaine aux Iles Canaries, sur Lanzarote - l'île aux 300 volcans. Six jours de randonnée dans un monde volcanique, avec une diversité dans la flore, les couleurs, les reliefs. J'y ai trouvé des enseignements frappants de géographie humaine - des illustrations de la manière dont l'humain s'est accommodé de son milieu et y a développé des façons de (sur)vivre. Mais j'aimerais ici dire comment je me suis senti "étranger», dans un groupe sympathique d'une douzaine de personnes dont la majorité avaient entre 35 et 55 ans. Par exemple en les voyant se précipiter sur leurs ordinateurs portables au retour de chaque activité. "Parce que, tu comprends, je reçois une centaine de mails par jour, il faut que je m'en occupe sans attendre.» Avec cela, les téléphones aux enfants et connaissances. Beaucoup regardent les paysages pour l'essentiel à travers leur smartphone.

Ma femme et moi avons la joie d'avoir de beaux petits-enfants. Ceux qui ont entre 10 et 16 ans passent énormément de temps sur leurs écrans et tablettes. Bon, ils sont de leur génération... Où j'ai été un peu amer l'été dernier, c'est de constater lors d'un voyage au sud de l'Europe comment ils peuvent rester toute une journée (ensoleillée) dans leur chambre de notre Airbnb, sans intérêt apparent pour les monuments ou les sites naturels de l'endroit visité. Quand je voyage, en train, en voiture ou en avion, une de mes "passions" est d'être à la fenêtre pour découvrir les régions traversées; les jeunes que je vois semblent ne pas imaginer qu'on puisse regarder par la fenêtre - et le grand-père se rend désagréable par ses remarques.

J'ai beaucoup randonné/trekké, je trouve de grandes satisfactions à marcher, spécialement loin de tout. Pour quelques jours ou même deux ou trois semaines, ma doctrine était «(si) pas de nouvelles, bonnes nouvelles» (j'envoie - encore - des cartes postales, qui arrivent après moi). Ici, je me promène souvent seul, y compris en montagne, et ai dû réaliser qu'on pouvait trouver critiquable d'être sans téléphone portable (quand j'y pense, j'emporte alors un Nokia «antique»). Un marcheur solitaire que j'ai lu regrettait qu'«on n'ait maintenant plus le droit de se perdre». Du côté positif, on pense bien sûr à ceux qui jusqu'il y a, disons, un quart de siècle ont perdu la santé, la vie ou le lien avec leurs proches parce qu'ils n'ont pu signaler où ils étaient (par exemple, histoire vraie du livre et film Into the Wild - ou, lors des migrations vers l'Amérique, ces familles dont un membre se perdait, sans pouvoir se retrouver jamais).

Aujourd'hui: ce qui frappe, c'est l'indispensabilité existentielle qui devient celle du smartphone - avec ses potentialités inédites d'avoir dans la main pratiquement toutes les connaissances existantes. Récemment, j'observais comment mes compagnons, d'une part, tapaient sur leur appareil dès qu'était mentionnée une question (plante, géographie, histoire) et, d'autre part, étaient véritablement "perdus» quand ils ne l'avaient pas sur eux. Le problème est bien proche du manque, de la dépendance.*

Implications pour la suite? Il ne peut être question d'arrêter l'évolution, mais de réfléchir (un peu quand même) au fait que, dans la vie quotidienne, nous de-

\section{Nos cinq sens seront moins finement utilisés ;} des senseurs multiples vont saisir la réalité mieux que nous.

venons des cyborgs, combinaisons homme-machine (y compris grâce aux merveilles de la médecine: pacemakers, greffes de matériaux artificiels - et votre serviteur avec deux prothèses de genoux dont il est très satisfait). Une conséquence, me semble-t-il, est que notre appréhension du monde, la connaissance de notre environnement, ne sera plus directe comme elle l'était depuis toujours, mais sera de plus en plus médiatisée par des auxiliaires technologiques. Logiquement (?), nos cinq sens seront moins utilisés, perdront de leur utilité (s'atrophieront?): à leur place, des senseurs multiples vont saisir la réalité extérieure, mais aussi notre réalité intérieure - paramètres biologiques - bien mieux que nous. Et ce ne sont pas seulement les sens qui perdront de leur importance, mais aussi la mémoire puisque google peut sans effort tout nous dire en un clic.

Suis-je complètement dans l'erreur? Je le souhaiterais. 